\title{
CONGESTION FREE CHANNEL ALLOCATION FOR HIGH TRAFFIC USING MAC IN WSN
}

\author{
V. M. Jemin', Dr. M. N. Saravana kumar ${ }^{2}$, Dr. M. Ayyadurai ${ }^{3}$, Dr. R. Ashok kumar ${ }^{4}$ \\ ${ }^{1}$ Assistant Professor, RMK College of Engineering and Technology, Chennai,vmjemin@gmail.com \\ ${ }^{2}$ Assistant professor, Erode Sengunthar Engineering College, Erode,dr.mnskphd@gmail.com \\ ${ }^{3}$ Assistant Professor, Saveetha School of Engineering, ayyaduraiece@gmail.com \\ ${ }^{4}$ Assistant Professor, GRT Institute of Engineering and Technology, Chennai,asho291085@gmail.com
}

\begin{abstract}
Slot Allocation Medium Access Channel (SAMAC), an efficient slot allocating MAC protocol is proposed for predicting the timely channel access for time-critical data operations and also to reduce data congestion. In this scheme the time of non-periodic critical traffic grabs the time slots allotted for the periodic non-critical data traffic. Additionally, Earliest Deadline First (EDF) scheduling algorithm is used to offer efficient channel access in emergency conditions where numerous sensor nodes are prompted concurrently to transmit the periodic time-critical data to the controller. SAMAC protocol is analyzed with the delay in data delivery rates for the time-critical traffic. Simulation analysis is carried out for proving the efficiency of SAMAC in terms of network throughput.

Keywords: Medium Access Channel, Earliest deadline first scheduler, Critical traffic, Non-critical timeliness traffic, Sensor Network.
\end{abstract}

\section{Introduction}

Wireless Sensor Network (WSN) consists of spatially distributed autonomous sensor nodes to monitor physical or environmental conditions, such as temperature, sound, pressure, etc. Sensors in WSN have sensing capability and forwarding the real-time data to Base Station (BS) that collects data through their alloted time-slot [1]. WSNs are getting wider application prospects for sensor nodes that can get new functions after updating program codes [2]. The sensors are powered up with batteries and frequent replacements of batteries are quite difficult in harmful areas. The energy is monitored using matrix completion technique [3]. Hence consumption of energy rate for node's should be minimized by avoiding unnecessary transmissions [4] and to reduce packet transmission delays duty cycle operations needs to be carried out in order to achieve a better network lifetime $[5,6]$.

Medium Access Channel (MAC) seems to be an effective layer and it has direct communication control ability that avoids unnecessary delays and energy consuming rate during network operations [7]. MAC layer highly helps in coordinating and node communication. MAC protocol design is highly based upon 'nodes and networks' energy consuming rate, data congestion and network lifetime.

\section{Related Works}

Several protocols were proposed for traffic aware routing with duty cycle operations in wireless network and some of them are discussed here. Sleep scheduling MAC (S-MAC) was proposed to reduce packet collisions and sleep/listen periods is allotted for nodes to maintain the overhearing issues [8]. In S-MAC, the listen phase is fixed and the sleep phase is fixed based upon a duty cycle factor i.e. application based. Listen phase is further divided into SYNC and Data periods. Exchange of data packets is occurred in data periods that include request-to-send, clear-tosend, DATA, and acknowledgment messages. To avoid latencies that occur due to sleep periods TMAC an adaptive listening scheme was proposed [9]. Forecast Function based Congestion Control in MANET Routing (FFCC). Here, highest congested node is measured using node congestion rate and isolates that highly congested node from routing. The less congested node is selected here so that the data reaches the destination without any loss [10].

In adaptive duty cycle mechanism traffic load, traffic priority and size of the queue are the parameters used to regulate the node's duty cycle. Latencies occurred due to packet transmission done using duty cycle that is taken care of using length based broadcast scheme by the delay encountered during sleep mode [11]. SElfAdaptive duty cycle MAC (SEA-MAC) was proposed using dual adaptive mechanisms that uses adaptive scheduling mechanism makes the nodes' active duration adaptive to variable traffic load, thus enabling SEA-MAC to resiliently schedule data transmission [12].

In DutyCon [13], the duty cycle is controlled by feedback controller and the delay is achieved along with efficiency in energy. The duty cycle is controlled using node's proportion of single-hop delay condition as well as the delay occurred due to packet transmission; obtained through timestamps. Here the duty cycle is adjusted dynamically using adaptive duty cycle scheme 
which is controlled through node's interval [14]. Energy Efficient and QoS-aware (EEQ) MAC (EEQMAC) protocol with a duty cycle mechanism adapts the node's duty cycle to the queue size and packet wise priority to minimize the higher priority packet delay that highly support time-bounded priority packets delivery[15]. This EEQMAC checks the class of traffic and queue size for each node in the message queue and determines the adjustments of duty cycle. The active period is increased in length if any high traffic event occurs that consequences in less waiting time for the packets that staying in queue.

\section{Proposed System: SAMAC}

SAMAC is a slot allocating MAC protocol proposed to predict the timely access of channels for time-critical data operations and also to reduce data congestion. Here non-periodic time-critical traffic grabs the time slots allotted from the periodic time-critical traffic. An Earliest Deadline First (EDF) scheduling algorithm is used to offer guaranteed channel access in emergency conditions and event-based situations where numerous sensor nodes are prompted concurrently to transmit the periodic time-critical data to the controller. Centralized control model using star network topology is taken here for the Industrial WSN. The system is modelled with a central controller (node acting as base station) and ' $n$ ' number of sensor nodes located at different locations to gather information. In this proposed SAMAC the network can hold up two types of traffic models such as $\mathrm{C}_{\mathrm{T}}$ traffic and $\mathrm{NC}_{\mathrm{T}}$ traffic. $\mathrm{C}_{\mathrm{T}}$ denotes data traffic of time-critical operations with unexpected causes like protection interlocking and emergency pack up. This traffic type is marked as critical and is set with (bounded up) earliest first deadlines. $\mathrm{NC}_{\mathrm{T}}$ denotes non-critical data traffic that includes cyclic process of monitoring. Here the ' $n$ ' number of nodes is segmented into two clusters; first cluster consists of ' $\mathrm{k}$ ' number of nodes which is responsible for transmitting ' $\mathrm{C}_{\mathrm{T}}$ ' data traffic in accordance with the certain emergency circumstances.

Second cluster comprises of ' $n-k$ ' $N$ balance number of nodes that certainly responsible for sending the ' $\mathrm{NC}_{\mathrm{T}}$ ' data traffic. Here a shortest range of distance communication circumstances along with an error-free channel (low congested nodes) is considered. The value of ' $k$ ' is identified in accordance to the information type i.e. any kind of emergency event is sensed in the industrial environment. Therefore the ' $\mathrm{k}$ ' number of nodes is located on the basis of critical environments and hence the precise information can be received in timely manner with the help of low congested nodes. The System model of SAMAC is shown in figure 1 .

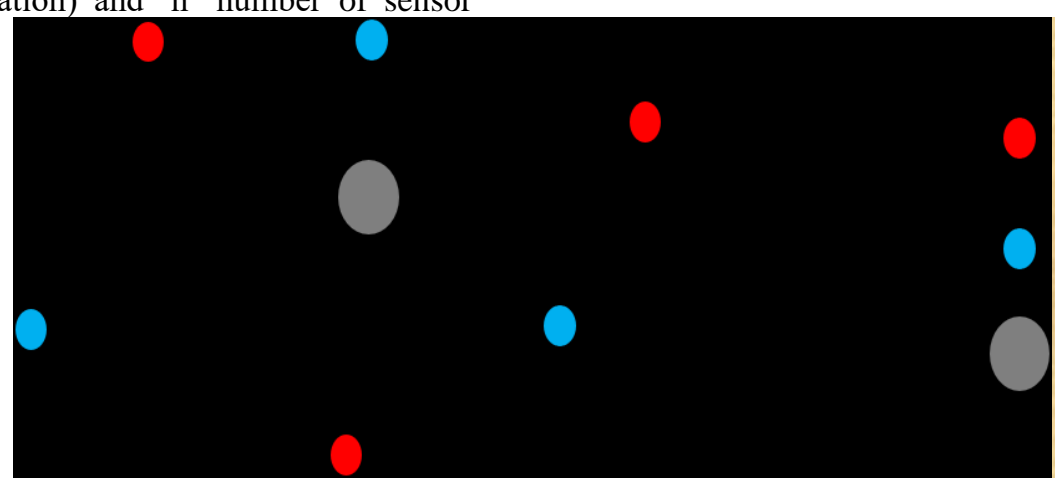

Figure 1: System Model of SAMAC

(i) Channel Access Method for $\mathrm{C}_{\mathrm{T}}$ Traffic

The data traffic in the first cluster which consists of ' $\mathrm{k}$ ' number of nodes with the critical timeliness data ' $\mathrm{Ct}$ ' and $\mathrm{BS}$ is carried out in the form of successive transmission cycles which is shown in figure 2 .

Figure 2: Transmission cycle of Pc traffic 
Each data cycle consists of a time slot with period $S_{\mathrm{T}}$ and an Emergency Signal Slot (ESS) with period $\mathrm{ESS}_{\mathrm{T}}$. The $\mathrm{ESS}_{\mathrm{T}}$ in each data transmitting cycle undergoes three phases includes Request for Reservation (RR) phase, Scheduling Phase (SP) and Transmission Phase (TP). Then the reservation phase can be further divided into ' $\mathrm{K}$ ' sub-slots with a period $\mathrm{S}_{\mathrm{TK}}$ for sending the request for channel access.

(ii) Channel Access for $\mathrm{NC}_{\mathrm{T}}$ Traffic

The traditional Time Division Multiple Access (TDMA) channel access scheme is considered for sending the non-critical data traffic from the second cluster to the BS and their timing structure is shown in the figure 3 . Each $\mathrm{NC}_{\mathrm{T}}$ node is allocated with the time slot of duration $S_{T}$ for transmitting the sensed data over the channel. A time duration of emergency slots $\mathrm{ESS}_{\mathrm{T}}$ is inserted between each successive time slots of $\mathrm{NC}_{\mathrm{T}}$ since the inserted slots can be used for the emergency cases to prioritize the data traffics of $\mathrm{C}_{\mathrm{T}}$ over the $\mathrm{NC}_{\mathrm{T}}$ data traffic. During each transmission of $\mathrm{NC}_{\mathrm{T}}$ traffic it checks the queue for any emergency traffic to be sent. If the queue slot is null, then the $\mathrm{NC}_{\mathrm{T}}$ node will process its transmission with its allotted time slot. If the channel queue slot is indicated with ESS with duration $\mathrm{ESS}_{\mathrm{T}}$ then the channel queue is set as busy with the parameter ' $\mathrm{x}$ ' so that $\mathrm{NC}_{\mathrm{T}}$ traffic waits till the channel status ' $x$ ' is set to ' 0 ' i.e. becomes null. The network is generally set up with $\mathrm{N}$ number of nodes, each node has its allocated time frame for sending the data over the TDMA channel with the $\mathrm{N}$ number of time-slots, $\{\mathrm{T} 1, \mathrm{~T} 2, \ldots, \mathrm{TN}\}$.

Figure 3: Timing structure for $P_{n c}$ Traffic

Algorithm: $C_{T}$ and $N_{T}$ traffic transmission

1: if $N_{i}$ has $\mathrm{C}_{\mathrm{T}}$ traffic to transmit then

2: Send add emergency sign $\mathrm{ESS}_{\mathrm{T}}$ in queue

ConstructingEDF:

3: Apply EDF to transmit $\mathrm{C}_{\mathrm{T}}$

4: Add RR with binary coded $d i$

5: Note for $\mathrm{NC}_{\mathrm{T}}$ in the channel

6: if $\mathrm{NC}_{\mathrm{Ti}}=0$ then

7: forward $\mathrm{C}_{\mathrm{T}}$ traffic to $\mathrm{BS}$

8: else

Queuing:

9: Add $\mathrm{x}=\mathrm{C}_{\mathrm{Ti}}$

10: while $x>0$ do

11: $\mathrm{x}=\mathrm{x}-1$

12: end while

13: Set $\mathrm{ESS}_{\mathrm{T}}$ with ' $\mathrm{x}$ '

14: forward $\mathrm{C}_{\mathrm{T}}$ traffic

15: Now set $x=0$ then

16: Send $\mathrm{NC}_{\mathrm{T}}$ traffic to $\mathrm{BS}$

17: else

18: gotoQueuing

19: end if

20: end else

Therefore by prioritizing the data traffic on basis of their critical timeliness highly reduces the packet loss and information latency. This process also reduces the energy consumption by reducing retransmissions in the channel.

\section{Results and Discussion}

The simulation analysis is carried out for the conventional and proposed schemes in an iterative order so that the results can be obtained with the maximum accuracy. The results of the proposed SAMAC and conventional EEQMAC are compared for their efficiency. The packets generated from the trace file are used for conducting the qualitative analysis. The parameters considered here for evaluation are average data traffic delivered, end-to-end delay for variable rates and variable nodes, and energy consumption to test the effectiveness of the SAMAC protocol with their analyzing metrics.

\section{a. Average data traffic delivered}

The ratio of average data rates that delivered to the base station with respect to the data traffic that sent by the sender node is said to be average data traffic delivered. It is measured using equation 1 .

$$
\text { Average_DR }=\frac{\sum_{0}^{n} \text { Packets Received }}{\text { Time }}
$$


Here, Average_DR represents average data traffic delivered and ' $n$ ' denotes number of nodes.

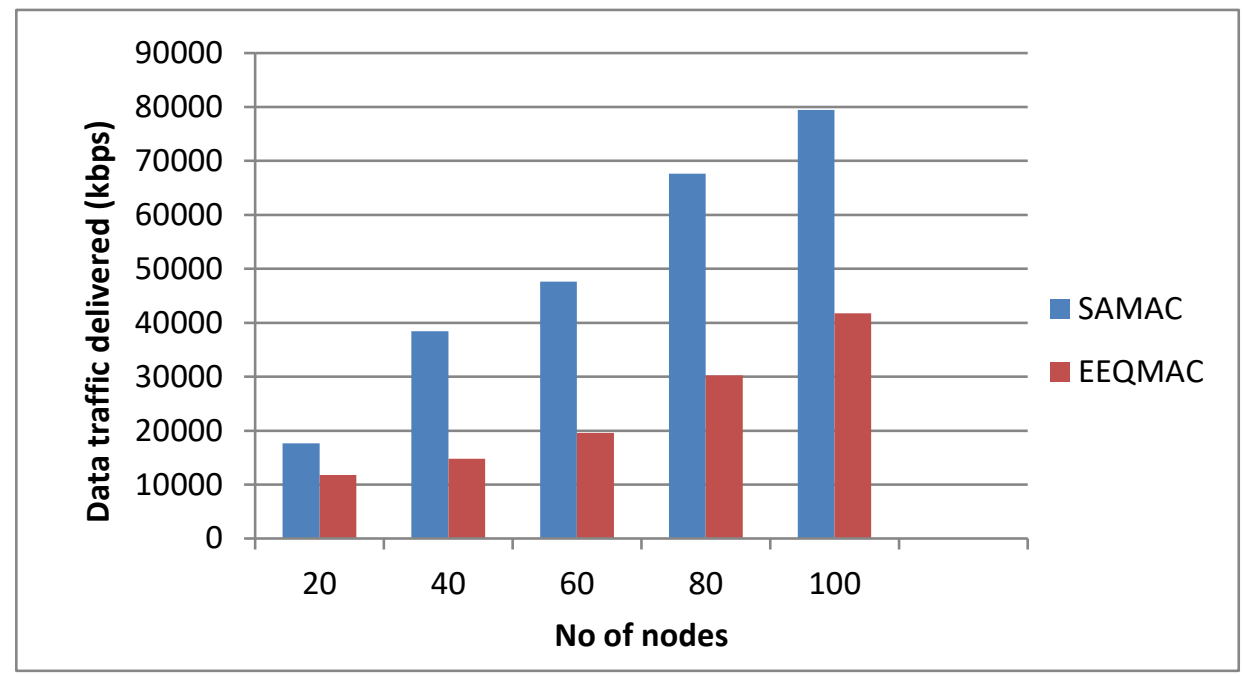

Figure 4: Average Data Traffic Delivered

Figure 4 shows the graphical representation of average data traffic delivered for the proposed and conventional scheme. Proposed SAMAC has high delivery rates of data traffics over the MAC channel compared to the conventional EEQMAC. b. $\quad$ End to End Delay (varying nodes)

The End To End (ETE) delay is defined as the time dissimilarities between the presently receiving data traffic packets and previous received data traffic. ETE is measured using equation 2.

$$
\text { ETE_Delay }=\frac{\sum_{0}^{n}(\text { Data Rcvd Time }- \text { Data Sent Time })}{n}
$$

Here $\mathrm{n}$ represents number of nodes.

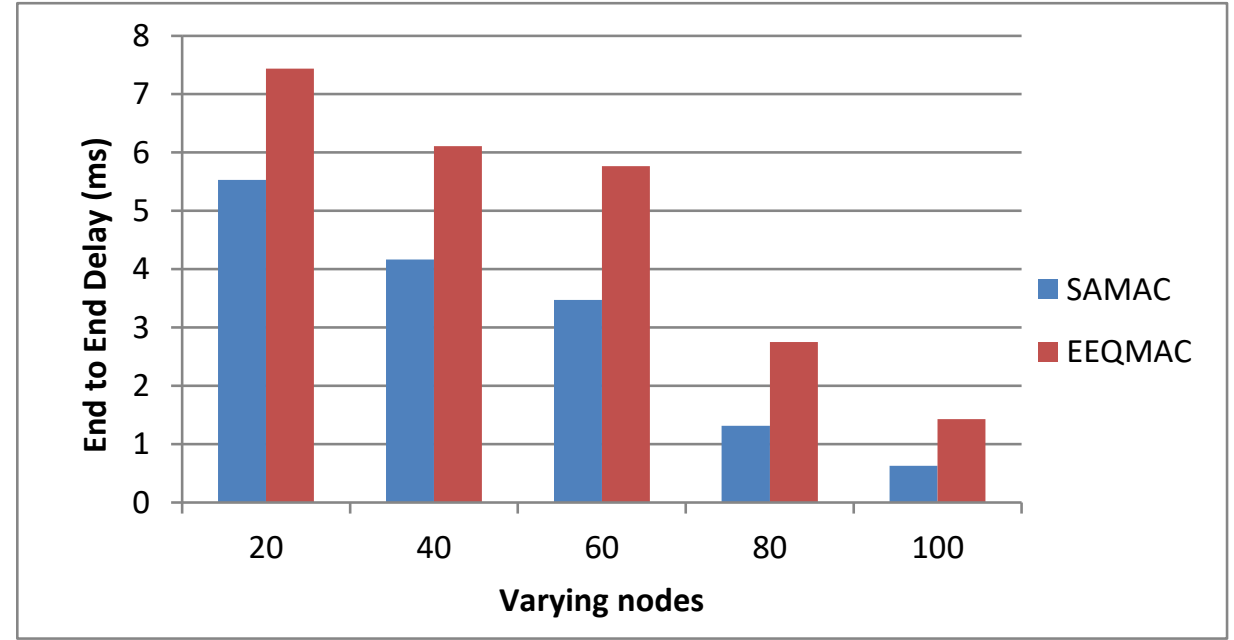

Figure 5: ETE Delay (varying nodes)

The ETE Delay for varying nodes is shown in figure 5. SAMAC has lesser delay values in processing the data traffic over the network compared to the EEQMAC protocol.

\section{c. ETE Delay (varying rates)}

The ETE delay is defined as the time difference for the sending and receiving data traffic for the single hop transmission in terms of varying rates. 


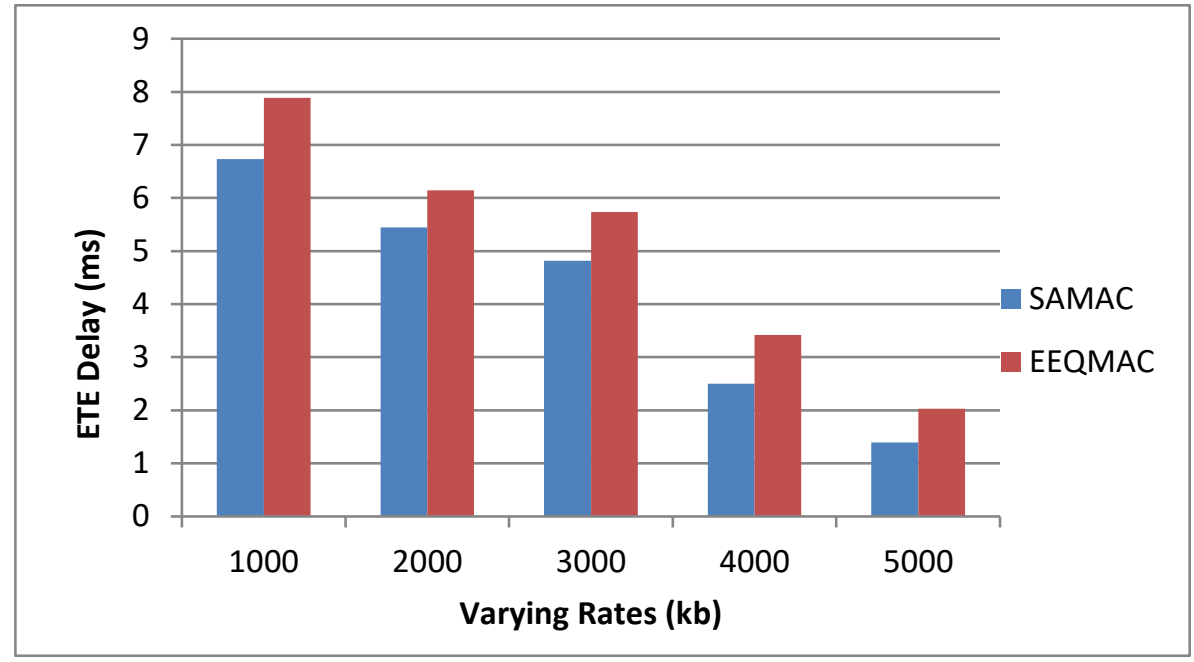

Figure 6: ETE Delay (varying rates)

Figure 6 shows the graphical representation of ETE delay with varying rates for both proposed SSMAC and conventional EEQMAC. The proposed scheme SAMAC has low end to end delay while comparing to their conventional scheme. Lower delay indicates that the network results in higher throughput.

\section{d. Energy Consumption}

The amount or the quantity of energy that is consumed by the node to process the transmission, receiving and sending the data traffic at the instance of time is defined as the process of energy consumption. Energy consumption calculation gives the rate of level of available energy for the further process of network operations.

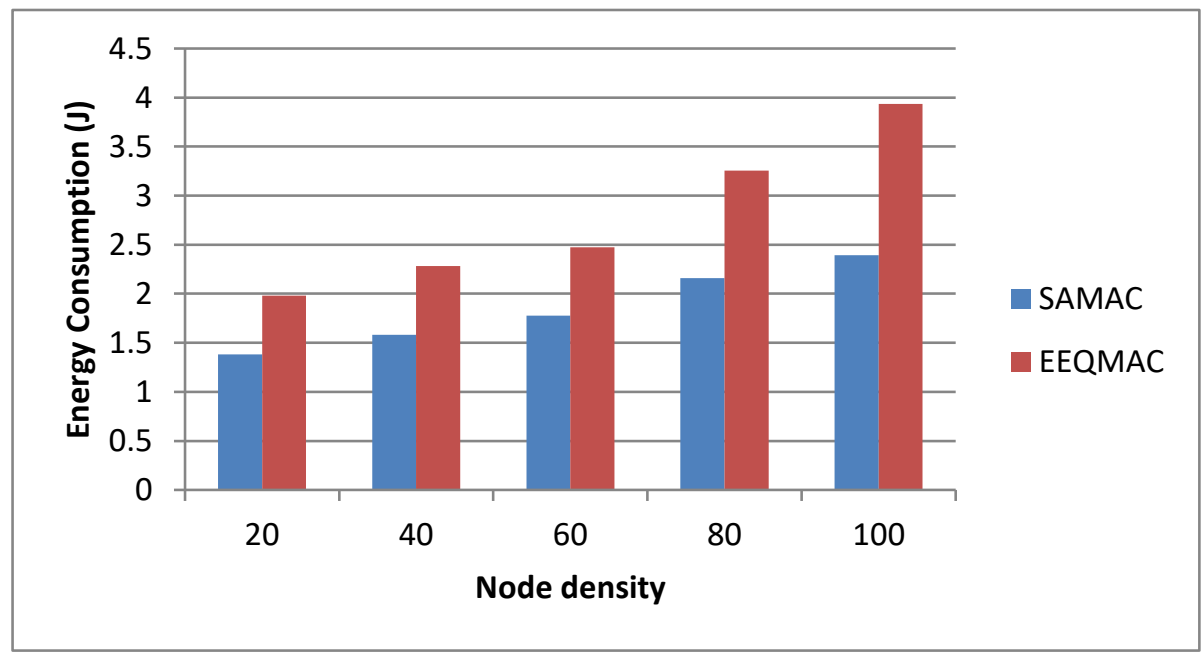

Figure 7: Energy Consumption

The comparison of energy consumption for both proposed SAMAC and existing EEQMAC is shown in figure 7. The EEQMAC scheme consumes more energy for processing the data traffic compared to the proposed SAMAC scheme.

\section{Conclusion}

To predict the timely access of channel for time-critical data operations and to reduce data congestion over the channel SAMAC is proposed. Here periodic time-critical traffic grabs the time slots allotted from the time of aperiodic non-critical traffic. EDF scheduling algorithm is applied to offer the free passing transmission channel access in case of emergency conditions where many are prompted concurrently to transmit the periodic time-critical data to the BS. The proposed protocol is evaluated with the ETE delay both varying rates and varying nodes in terms of data transmission rates for the time-critical traffic. Simulation results are analysed to show the efficiency of the proposed model. 


\section{References}

1. Liu, Q., \& Liu, A. (2018). On the hybrid using of unicast-broadcast in wireless sensor networks. Computers \& Electrical Engineering, 71, 714-732. 2.Yu, S., Liu, X., Liu, A., Xiong, N., Cai, Z., \& Wang, T. (2018). An adaption broadcast radiusbased code dissemination scheme for low energy wireless sensor networks. Sensors, 18(5), 1509.

3. Tan, J., Liu, W., Xie, M., Song, H., Liu, A., Zhao, M., \& Zhang, G. (2019). A low redundancy data collection scheme to maximize lifetime using matrix completion technique. EURASIP Journal on Wireless Communications and Networking, 2019(1), 1-29.

4. Wang, F., Liu, W., Wang, T., Zhao, M., Xie, M., Song, H., ... \& Liu, A. (2019). To reduce delay, energy consumption and collision through optimization duty-cycle and size of forwarding node set in WSNs. IEEE Access, 7, 55983-56015.

5. Li, Q., Liu, A., Wang, T., Xie, M., \& Xiong, N. N. (2019). Pipeline slot based fast rerouting scheme for delay optimization in duty cycle based M2M communications. Peer-to-Peer Networking and Applications, 12(6), 1673-1704.

6. Yetgin, H., Cheung, K. T. K., El-Hajjar, M., \& Hanzo, L. H. (2017). A survey of network lifetime maximization techniques in wireless sensor networks. IEEE Communications Surveys \& Tutorials, 19(2), 828-854.

7. Muzakkari, B. A., Mohamed, M. A., Kadir, M. F., Mohamad, Z., \& Jamil, N. (2018). Recent advances in energy efficient-QoS aware MAC protocols for wireless sensor networks. International Journal of Advanced Computer Research, 8(38), 212-228.

8. Sherazi, H. H. R., Grieco, L. A., \& Boggia, G. (2018). A comprehensive review on energy harvesting MAC protocols in WSNs: Challenges and tradeoffs. Ad Hoc Networks, 71, 117-134.

9. Li, H., Jaggi, N., \& Sikdar, B. (2011). Relay scheduling for cooperative communications in sensor networks with energy harvesting. IEEE Transactions on Wireless Communications, 10(9), 2918-2928.

10. Suresh, G., Kumar, S., Kavitha, V., \& Lekashri, S. (2020, September). Forecast Function Based Congestion Control in MANET Routing. In IOP Conference Series: Materials Science and Engineering (Vol. 925, No. 1, p. 012074). IOP Publishing.

11. Shu, T., Liu, W., Wang, T., Deng, Q., Zhao, M., Xiong, N. N., ... \& Liu, A. (2019). Broadcast based code dissemination scheme for duty cycle based wireless sensor networks. IEEE Access, 7, 105258-105286.

12. Zhao, Y. Z., Miao, C. Y., \& Ma, M. (2013). An energy-efficient self-adaptive duty cycle MAC protocol for traffic-dynamic wireless sensor networks. Wireless personal communications, 68(4), 1287-1315.
13. Wang, X., Wang, X., Xing, G., \& Yao, Y. (2010, June). Dynamic duty cycle control for endto-end delay guarantees in wireless sensor networks. In 2010 IEEE 18th International Workshop on Quality of Service (IWQoS) (pp. 1-9). IEEE.

14. Byun, H., \& Yu, J. (2012). Adaptive duty cycle control with queue management in wireless sensor networks. IEEE Transactions on Mobile Computing, 12(6), 1214-1224.

15. Muzakkari, B. A., Mohamed, M. A., Kadir, M. F., \& Mamat, M. (2020). Queue and priority-aware adaptive duty cycle scheme for energy efficient wireless sensor networks. IEEE Access, 8, 1723117242. 